\title{
Computação em Nuvem para Gerenciamento de Rede de Ensino
}

\author{
Daniel Ferreira de Oliveira, Tarcísio Antonio Valentim Junior, Neide dos Santos
}

Departamento de Informática e Ciência da Computação

Universidade do Estado do Rio de Janeiro

danielfeol@gmail.com, danielfeol@gmail.com, neide@ime.uerj.br

\begin{abstract}
Resumo. A computação em nuvem refere-se à utilização da memória e das capacidades de armazenamento e processamento de computadores $e$ servidores compartilhados e interligados por meio da Internet, seguindo o princípio da computação em grade. Com o objetivo de experimentar um processo de desenvolvimento de uma cloud computing, este artigo apresenta SISESC (Sistema de Gerenciamento de Rede de Ensino), que utiliza os conceitos desta nova abordagem e que visa garantir a escalabilidade, a velocidade e a segurança aos usuários deste sistema. SISESC utiliza a computação em nuvem como sistema legado em que todos os recursos necessários estão disponíveis na "nuvem". Este artigo descreve a criação da nuvem, as etapas de desenvolvimento do protótipo SISESC e sumariza as formas de avaliação das aplicações em nuvem.
\end{abstract}

\begin{abstract}
Cloud computing refers to the use of memory and the capabilities of storage and processing of computer and shared servers interconnected via the Internet according to grid computing paradigm. In order to experience the cloud computing development process, this paper presents SISESC (School Network Management System) that uses this new development approach and aims to ensure scalability, speed and security to system users. SISESC uses cloud computing as legacy system in which all necessary resources are available in the "cloud". This paper describes the cloud building process; the stages of SISESC prototype development and briefly argues the ways of evaluating cloud computing applications.
\end{abstract}

\section{Introdução}

O conceito de computação em nuvem (em inglês, cloud computing) refere-se à utilização da memória e das capacidades de armazenamento e processamento de computadores e servidores compartilhados e interligados por meio da Internet, seguindo o princípio da computação em grade. A computação em nuvem oferece uma solução interessante para o desenvolvimento de software e acesso de conteúdo com a transparência da localização da infra-estrutura utilizada. A infra-estrutura da nuvem é geralmente composta por vários datacenters em que os consumidores têm acesso a apenas uma parte do poder computacional de uma rede escalável. A prestação desses recursos computacionais é controlada por um provedor, e os recursos são alocados em uma maneira elástica, de acordo com as necessidades dos consumidores. 
A maior vantagem deste modelo é utilizar, em qualquer lugar e independentemente de plataforma, as mais variadas aplicações por meio da Internet com a mesma facilidade de tê-las instaladas em nossos próprios computadores. $\mathrm{O}$ uso das nuvens como um tipo de infra-estrutura para software em execução é bastante diferente das práticas tradicionais, onde o software é executado sobre infra-estruturas, muitas vezes dimensionadas de acordo com o uso do pior caso. Para acomodar demandas imprevistas de forma escalável e elástica, o processo de alocação e realocação em computação em nuvem deve ser dinâmico. Outra característica essencial dos mecanismos de alocação de recursos em computação em nuvem é garantir que os requisitos de todas as aplicações sejam devidamente atendidos.

Para alcançar este requisito, qualquer mecanismo de alocação em computação em nuvem deve estar ciente do estado de cada elemento / recurso na infra-estrutura. Então, o mecanismo deve aplicar algoritmos para alocar melhor os recursos físicos ou virtuais para aplicações dos consumidores, de acordo com os requisitos préestabelecidos com a nuvem provedor.

Além do benefício dos serviços elásticos, computação em nuvem permite que os consumidores reduzam ou eliminem custos associados com a infra-estrutura interna para a prestação dos seus serviços. Esta oportunidade de redução de custos faz dela uma alternativa atraente, especialmente para iniciativas de negócios. ARMBRUST et al (2009) sugerem que este modelo de benefícios é uma forma de multiplexação, uma vez que aloca recursos para vários usuários simultaneamente. A multiplexação de datacenters é garantida através de várias décadas de pesquisa em áreas como a computação em grade, os sistemas distribuídos, as tecnologias web, a computação de serviços e a virtualização.

O entusiasmo com a computação em nuvem vem justamente das inúmeras vantagens que o modelo oferece, tanto aos fornecedores de tecnologia quanto aos usuários, e uma das vantagens é o melhor aproveitamento dos investimentos em hardware e softwares, proporcionando economia de custos. Não há a necessidade de investimentos maiores em equipamentos potentes na ponta para acessar as aplicações, ou de trocar componentes ou até equipamentos inteiros para fazer upgrade de capacidade de processamento, por exemplo. Como a computação em nuvem oferece flexibilidade maior, basta que o usuário final tenha um navegador e uma boa conexão à Internet, já que a parte mais pesada do processamento fica na nuvem.

Diante das revoluções tecnológicas e do advento da web 2.0 e da computação em nuvem, surgem cada vez mais empresas interessadas em expandir seus serviços a esta nova tendência.

Atualmente, a maioria das empresas utiliza pesados e relativamente caros servidores que gerenciam e dão suporte a uma rede de no mínimo 100 computadores conectados a eles. Vários são os custos, entre eles a necessidade de se ter hardware robusto que dê suporte ao banco de dados e ao sistema operacional que fará o gerenciamento, de se instalar sistemas funcionais nas máquinas clientes e eventualmente de se fazer manutenções e/ou atualizações, além dos custos com licenciamento de softwares, entre outras. 
Com o objetivo de experimentar um processo de desenvolvimento de um provedor de computação em nuvem, neste projeto é apresentado SISESC - Sistema de Gerenciamento de Rede de Ensino, que utiliza os conceitos desta nova abordagem e que visa garantir a escalabilidade, a velocidade e a segurança aos usuários deste sistema. Em redes de ensino, os custos em manter um bom sistema de gestão são os mesmos. SISESC utiliza a computação em nuvem como sistema legado em que todos os recursos necessários estão disponíveis na nuvem.

Com o propósito de apresentar o sistema proposto, este artigo está organizado em três seções, além desta introdução: a seção 2 oferece uma visão geral de computação em nuvem; a seção 3 descreve a criação da nuvem, as etapas de desenvolvimento do protótipo SISESC e sumariza as formas de avaliação das aplicações em nuvem; e, por fim, a seção 4 oferece as conclusões do estudo e as perspectivas futuras.

\section{Computação em Nuvem: Visão Geral}

Nesta seção, é apresentado o conceito de computação em nuvem e detalhado Eucalyptus, um framework para a implementação de um serviço de computação em nuvem do tipo IaaS (Infrastructure as a Service).

A computação em nuvem oferece uma solução interessante para os desenvolvedores de sistemas e acesso ao conteúdo por parte dos clientes finais de forma transparente quanto à infra-estrutura utilizada para fornecimento dos serviços. Sua infra-estrutura é composta de um ou vários datacenters, dependendo do fornecedor do serviço. Não há consenso entre os teóricos sobre o que é ou não ser computação em nuvem, visto que muitas empresas já forneciam alguns de seus serviços bem antes do conceito existir. Por ser um conceito novo na área de Tecnologia da Informação (TI) também não existe consenso sobre a melhor definição, especialmente no meio acadêmico. Devido à complexidade e contemporaneidade do tema, se torna relevante discutir brevemente o conceito.

Para BUYYA et al (2008), a computação em nuvem é um tipo de sistema paralelo e distribuído que consiste em uma coleção de computadores interconectados e virtualizados, que são dinamicamente provisionados e apresentados como um ou mais recurso computacional unificado. Tais recursos têm que ser baseados em acordos de níveis de serviço (SLA - Service Level Agreement) estabelecidos através de negociações entre o provedor de serviços e os consumidores. Para FOSTER et al (2008), a computação em nuvem pode ser definida como um paradigma de computação distribuída que é impulsionado pelas economias de escala, na qual um conjunto gerenciável de poder computacional, de armazenamento e plataformas consideradas abstratas, virtualizadas e dinamicamente escaláveis são entregues sob demanda para clientes externos através da Internet. Essa definição é importante, pois demonstra que esse novo conceito difere dos demais paradigmas computacionais por concentrar pontos chave, como o fato de ser escalável, por ter a característica de oferecer diferentes níveis de serviços aos clientes, por ser motivado pela economia de escala e, por fím, pela observação que esses serviços podem ser configurados dinamicamente e entregues sob demanda.

Já a definição da consultoria americana Gartner Group diz que computação em nuvem é um estilo de computação escalável e elástica, na qual os recursos de TI são 
fornecidos como um serviço para clientes externos a partir da internet. A empresa define ainda cinco atributos que caracterizam a computação em nuvem (GARTNER, 2009):

(1) Ser baseado em serviço - A solução deve ser implementada como um serviço e o que ele foi designado a fazer deve ser mais importante do que a forma como as tecnologias são usadas para disponibilizá-lo. O serviço deve ter sido projetado para atender às necessidades específicas de um conjunto de consumidores, e as tecnologias são adaptadas a essa necessidade, podendo ser consideradas sob medida;

(2) Ser "escalável" e elástico - O serviço deve poder aumentar ou diminuir sua capacidade (escalar) de acordo com as exigências dos consumidores, na velocidade que eles precisam, o que pode consumir alguns segundos ou horas. Já a elasticidade está associada não só com escala, mas também a um modelo econômico que permite essa escalabilidade em ambos os sentidos de uma forma automatizada;

(3) Ser compartilhado - O serviço deve partilhar um conjunto de recursos a fim de atingir a economia de escala. Os recursos de TI devem ser usados com o máximo de eficiência, sendo divididos entre os diversos usuários dos serviços. Isso permite que recursos não utilizados sejam realocados para os clientes que mais precisam;

(4) Ser medido por uso - O serviço deve ser monitorado através do uso de métricas que permitam diferentes modelos de pagamentos. Esses modelos serão baseados no uso do serviço e não sobre o custo do equipamento e terão por base os serviços utilizados pelos consumidores, que podem ser mensurados em horas, transferências de dados, entre outros;

(5) Usar tecnologias da Internet - O serviço deve ser oferecido através de protocolos e formatos web (como URLs, HTTP e IP), o que o caracterizará como um serviço remoto.

Através dos conceitos apresentados, pode-se considerar que computação em nuvem é um serviço computacional oferecido através da Internet de acordo com a necessidade do cliente por um provedor especializado. É importante entender essa conceituação por que o valor e a originalidade da computação em nuvem estão justamente em embalar e oferecer recursos de um modo econômico, escalável e flexível, que é acessível e atrativo para toda empresa que deseja reduzir seus custos e ter a possibilidade de transferi-los ao desenvolvimento de seu core business.

Vale lembrar também que quando nos referimos aos agentes envolvidos na computação em nuvem, estamos nos referindo, segundo (VAQUERO et al. 2008), aos três principais: clientes, desenvolvedores e provedores. Notando que o provedor é o agente que vai lidar com dois tipos de usuários, clientes e desenvolvedores. Em computação em nuvem, usuários desenvolvedores criarão novos serviços que serão acessados pelos usuários clientes. O cliente é o consumidor do serviço produzido pelo desenvolvedor.

Os desenvolvedores podem ser programadores independentes dos provedores e trabalhar em cima da transparência de localidade fornecida pelos provedores, não sendo necessário a priori nenhum conhecimento da arquitetura da empresa contratada como provedora. A Figura 1 apresenta uma abstração do cenário típico de agentes em uma computação em nuvem. 


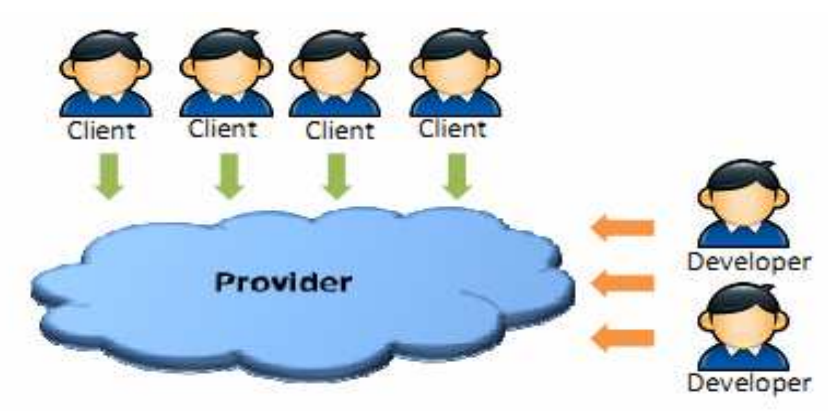

Figura 1. Agentes típicos da computação em nuvem

\subsection{Características da Computação em Nuvem}

A adoção do epíteto computação em nuvens é atribuída a uma metáfora para a Internet, uma abstração para a complexa infra-estrutura que ela esconde para poder funcionar (LAMB, 2009). Na verdade, esse novo paradigma emergiu como um desenvolvimento natural e de uma integração de avanços em diversos campos como a computação utilitária, SOA (Service Oriented Architecture), computação em grade, serviços Web e computação distribuída. Existem diversos serviços que são atrelados ao conceito e que podem trazer benefícios à operação das empresas, uma vez que podem ser adquiridos na quantidade necessária para cada caso (MOTAHARI-NEZHAD et al, 2009):

- PaaS (Platform as a Service): serviços para suportar todo o ciclo de vida de desenvolvimento de aplicações incluindo concepção, execução, debugg, teste, implantação, operação e apoio às aplicações Web e serviços na Internet;

- DaaS (Database as a Service): banco de dados normalmente não relacional para as aplicações das empresas, sejam internas ou rodando nas nuvens;

- IaaS (Infrastructure as a Service): recursos de hardware, como espaço para armazenamento de dados e capacidade de processamento;

- SaaS (Software as a Service): aplicações de software oferecidas como serviços na Internet.

Ao contrário do modelo tradicional, onde o cliente deve adquirir as licenças e instalar a aplicação em todos os seus equipamentos, aqui ela esta disponível através de um navegador, em qualquer lugar e a qualquer hora.

Nesse serviço se encaixa o oferecimento de sistemas SISESC para as escolas. Existem ainda outros tipos de serviços oferecidos pela computação em nuvem como gerenciamento, monitoramento e integração. No monitoramento como serviço, um fornecedor cuida das aplicações contratadas como serviço (SaaS) ou da rede de computadores de uma empresa em nome de seu cliente, e relata as métricas de desempenho ao seu contratante. O gerenciamento como serviço também inclui o monitoramento dos recursos computacionais, mas também tem a característica de agir quando necessário e não tão somente reportá-los.

Já na integração como serviço o objetivo é prover todo o ambiente para que se possa utilizar a plataforma como serviço (PaaS), permitindo o desenvolvimento de adaptadores e mapeamentos que proporcionem a interoperação de dados ou serviços heterogêneos. Ainda na idéia de oferecer as necessidades da empresa como serviço, 
outra importante atividade é a utilização de recursos humanos como serviço. Na verdade, a contratação de trabalhos esporádicos, através da Internet, remunerados somente pela atividade desejada não é algo novo no ambiente empresarial. Em atividades de TI muitas empresas já utilizam as habilidades de programadores ou analistas através da web, realizando o pagamento por horas trabalhadas ou atividade concluída. $\mathrm{O}$ que existe de diferente nessa utilização pela computação nas nuvens é o fato de os recursos utilizados serem especialistas em SaaS ou PaaS, e oferecerem consultoria para as empresas que desejam utilizar ou customizar o uso desses softwares como serviço ou integrar diversos desses aplicativos que rodam através da Internet para atingir os propósitos da corporação (MOTAHARI-NEZHAD et al, 2009). Com uma gama de diferentes atividades sobre o escopo de computação em nuvem, todos eles identificados como serviços disponíveis através da Internet para a contratação de acordo com a necessidade empresarial, esse novo paradigma cobre diversas tarefas necessárias à operacionalização de um negócio e tem o potencial de alterar as relações existentes nesse ambiente.

No modo tradicional de alocação de recursos computacionais pelas empresas é necessária à aquisição de equipamentos e infra-estrutura para todas as unidades de negócio, o que gera custos elevados e subutilização de equipamentos. A fim de tentar minimizar esse efeito, muitas companhias criaram sua própria infra-estrutura para distribuir de forma mais inteligente os recursos, ou seja, centralizaram sua base computacional. Nesse tipo de computação em nuvem, os dados e os processos são gerenciados pela corporação, que fica responsável por disponibilizar os serviços na quantidade pretendida para cada unidade. Conhecida como private cloud (nuvem privada), sua desvantagem é o alto investimento financeiro necessário para criar o ambiente que será utilizado, bem como os serviços que serão fornecidos. O outro tipo de nuvem existente é a public cloud (nuvem pública), que se caracteriza por estar disponível para uso através de um provedor de serviços e acessada a partir da Internet. É necessária a atenção ao fato de que o termo público não significa de graça, embora possam ser encontrados alguns serviços sem custo ou extremamente baratos. Por fim, o terceiro tipo de classificação para uma nuvem é a hybrid cloud (nuvem híbrida), que é a combinação de elementos das nuvens públicas e privadas. Esse tipo de nuvem é implementada quando se utiliza os serviços de nuvens públicas para lidar com as sobrecargas de sua nuvem interna. Assim, quando a estrutura criada pela empresa não suportar a utilização por causa de picos de trabalho, o serviço externo é contratado e disponibilizado aos usuários (IBM, 2009a).

Os três tipos de nuvem aqui apresentados podem ser utilizados em um projeto de disponibilização do sistema SISESC através de cloud computing, mas fica claro que as vantagens máximas da utilização do conceito serão advindas especialmente da implementação via nuvem pública. No caso especifico do desenvolvimento do presente trabalho, utilizaremos a nuvem sob o modelo PaaS (Platform as a Service), que fornece suporte ao desenvolvedor para todo ciclo de vida do projeto do sistema, inclusive fornecendo diversos frameworks ou sistemas mediadores, para tal fim, tais como Eucalyptus, XPC, Nimbus, OpenNebula, Enomaly e MapReduce. Nosso desenvolvimento será baseado no Eucalyptus, que é um framework open-source. 


\subsection{Framework Eucalyptus}

Elastic Utility Computing Architecture for Linking Your Programs to Useful Systems (EUCALYPTUS) é um framework desenvolvido em código aberto, que visa fornecer à comunidade científica uma plataforma para desenvolver experimentos e estudos de computação em nuvem. Ele foi desenvolvido inicialmente para prover serviços compatíveis com a nuvem da Amazon e possui interface totalmente compatível com software de código aberto para nuvem e soluções empresariais fornecidas pela Amazon. Sua arquitetura prevê duas classes de usuários diferentes, administradores e desenvolvedores. Os desenvolvedores podem, através do framework, requisitar e fazer uso de instâncias de maquinas virtuais, sem necessidade da intervenção manual do administrador, facilitando o aproveitamento da elasticidade desejada em uma computação em nuvem. Ela emprega serviços Web em toda sua comunicação entre as camadas de mediação do sistema. Possui quatro componentes (NURMI et al. 2008) conforme Figura 2: Maquinas Virtuais (VM) em um servidor, diretamente gerenciadas pelo controlador do nó (NC) em execução no mesmo servidor; Cluster Controller (CC) reunindo informações do estado de cada um dos NCs agrupados em clusters; no topo da arquitetura está o Cloud Controller (CLC); e, Walrus um serviço de armazenamento de dados compatível com a nuvem da Amazon.

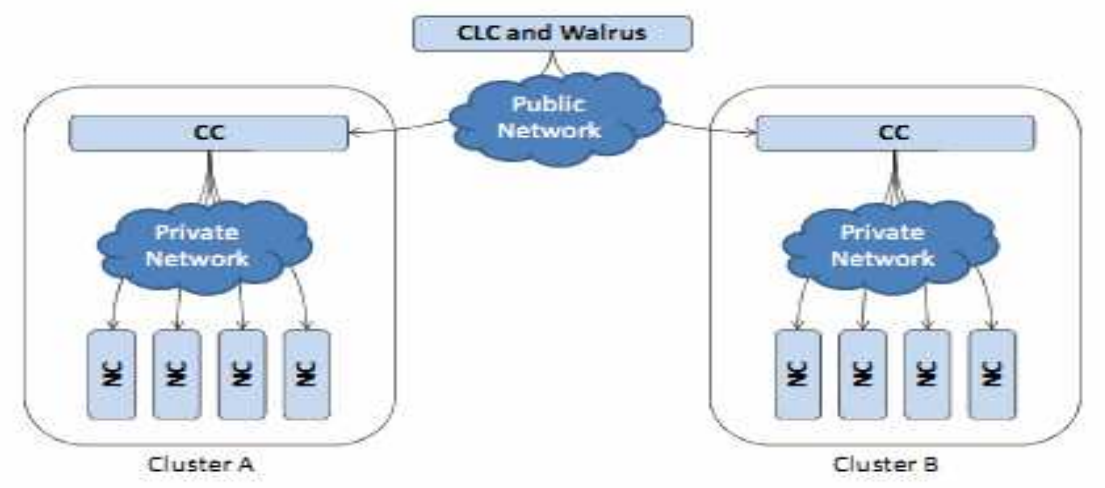

Figura 2. Componentes da arquitetura do Eucalyptus

Uma clara vantagem da arquitetura do Eucalyptus é seu ajuste natural para os ambientes de processamento existentes nas empresas, como resultado de seu design hierárquico. Foi desenvolvido inteiramente dentro do paradigma orientado a serviço. Portanto, mensagens trocadas entre seus componentes, bem como as mensagens enviadas por desenvolvedores que requerem um recurso são descritas através de interfaces de Web Services Description Language (WSDL). Em outras palavras, emprega Serviços Web para a comunicação em todas as camadas da mediação do sistema.

O gerenciamento de recursos no Eucalyptus está relacionado com a alocação e desalocação de máquinas virtuais nos servidores. Tal processo segue um algoritmo no qual a troca de mensagens é mostrada na Figura 3. O processo de alocação de recursos é desencadeado por um pedido do desenvolvedor e finalizado quando a VM está atualmente em execução em um nó (NURMI et al. 2008). Quando a interface de negociação sobre Eucalyptus recebe um pedido runInstances de um desenvolvedor, o CLC determina qual CC pode apoiar a VM por meio de consulta CCs através da 
operação describeResources e, em seguida, escolhendo o primeiro $\mathrm{CC}$ que responde com recursos livres suficientes. Da mesma forma, quando um CC recebe um pedido runInstances do CLC ele consultará cada NC através de mensagem describeResource e escolhe o primeiro NC que tem recursos livres suficientes.

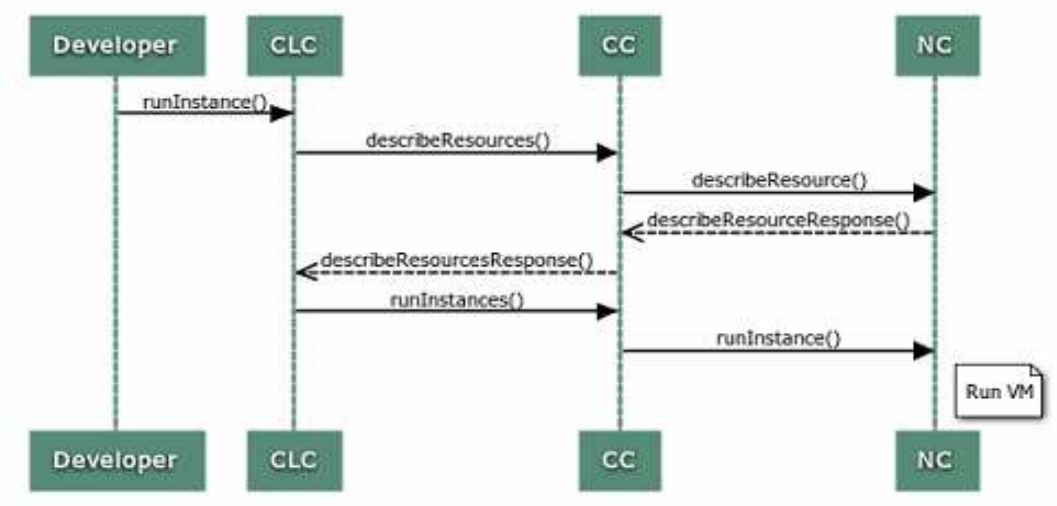

Figura 3. Diagrama de sequência de mensagens para executar uma VM

Considerando a potencialidade do conceito de computação em nuvem, foi desenvolvido um protótipo de sistema para gerenciar redes de escola. O domínio dos sistemas escolares foi escolhido por se considerar que as redes de ensino podem não dispor de poder computacional instalado em suas dependências para armazenamento e processamentos de suas informações.

\section{Sistemas de Gerenciamento de Redes de Ensino em Computação em nuvem}

No esforço de reduzir os custos com a implantação e manutenção dos sistemas de gerenciamento de redes de ensino, a idéia de poder utilizar e gerenciar o software como serviço, proposta pelo cloud computing, se torna um atrativo pelos ganhos que pode proporcionar. Ao se analisar os benefícios da adoção de um sistema SISESC através da nuvem de computação, a primeira vantagem percebida é a de utilizar o sistema diretamente da Internet, dispensando a necessidade de instalações e correções. Para fazer uso do software a escola precisaria somente de um browser que permita a navegação na Internet e também de uma assinatura do serviço de banda larga, requisitos comuns no ambiente empresarial atualmente.

A escola não precisará implantar e gerenciar toda a estrutura necessária para disponibilizar o aplicativo para seus colaboradores, e também não se preocupará em saber quantos servidores executam seu sistema, quais as configurações de hardware utilizadas, se são suficientes para sua demanda e como o escalonamento desses recursos computacionais é feito. Ela terá o aplicativo rodando na nuvem mesmo que sua infraestrutura seja extremamente simples, somente com computadores pessoais. Aqui já se percebe uma provável grande economia no investimento de recursos computacionais, visto que a implantação desse sistema no modelo tradicional demanda a compra de servidores de grande capacidade, máquinas robustas para cada usuário e licenças do programa e do banco de dados que serão utilizados. Seria possível, neste caso, o uso dos computadores conhecidos como "Thin clients", computadores sem grande capacidade 
de processamento e com custos baixíssimos. Também por essa vantagem, elimina-se a necessidade de sistemas operacionais (SO) específicos, trazendo a alternativa da implantação de SO's com código aberto (open source) que são gratuitos e podem ser instalados em todos os computadores da empresa sem restrições, ao contrário dos que são licenciados para uso em determinado número de equipamentos.

Outra potencial vantagem é a possibilidade de se controlar os gastos com o aplicativo de uma maneira mais flexível. Quando contratar o serviço de software ou os demais necessários para sua operação, a empresa saberá que o pagamento será realizado de acordo com a utilização, o que diminui o desperdício, visto que em muitas companhias os equipamentos e o software são subutilizados. Normalmente a companhia adquire um equipamento com uma capacidade muito maior do que a necessária, no intuito de se prevenir contra alterações de sistemas no futuro ou simplesmente para dar conta de picos de utilização. Com o uso de computação em nuvem quando a necessidade por recursos crescer por causa de uma grande quantidade de matrículas em suas unidades, ou por um período sazonal, basta contratar mais um pouco de serviço do fornecedor.

Outro ponto relevante é a característica do novo paradigma facilitar o compartilhamento de dados e o trabalho colaborativo entre as unidades da escola. Todos os dados necessários se encontram em um só lugar e com o mesmo formato, o que elimina a necessidade de conversões e adaptações. Se uma unidade e seu operador logístico, por exemplo, utilizarem o mesmo sistema SISESC nas nuvens, não haverá a necessidade de troca de mensagens, criação da infra-estrutura e integração entre aplicativos.

Os dados estarão disponíveis em tempo real e com um custo muito inferior ao que se tem hoje. Têm-se ainda prováveis benefícios relacionados ao trabalho executado pela equipe de TI da escola, que não necessitará mais se preocupar com procedimentos de backup dos dados e com o controle de segurança para acesso as informações. A manutenção, atualização e help desk para auxílio de usuários quanto ao sistema também não fariam mais parte de suas atribuições. Todas essas tarefas são executadas e de responsabilidade do provedor de serviço contratado, o que naturalmente trará uma revisão e reestruturação do departamento de TI que atua para a escola, seja este terceirizado ou composto de funcionários da própria empresa.

Por fim outra possível vantagem relativa à diminuição de custos está no decréscimo de utilização de energia elétrica, visto que o parque de máquinas poderá ser reduzido substancialmente e, consequentemente, o consumo também o será. A energia que seria consumida na escola com a manutenção dos equipamentos, muitas das vezes ociosos, passaria a ser consumida pelo provedor, com a diferença que o provedor possui melhores mecanismos de gerenciar seu gasto de energia, já que este é um dos desafios da computação em nuvem e varias idéias já existem em torno desse problema. Em tempos de preocupação com o meio ambiente e de busca da preservação dos recursos naturais, esse é um forte apelo para adoção da computação em nuvem pelas empresas, embora a redução de custos proporcionada pelo conceito seja a razão principal do interesse contemporâneo pelo tema.

É certo, portanto, que este novo paradigma computacional traz a possibilidade de ganhos substanciais para as empresas, tanto operacionalmente quanto financeiramente. 
Porém existem diversas ponderações sobre as desvantagens que a computação em nuvens pode acarretar no ambiente corporativo que devem ser levadas em conta pelos tomadores de decisões. ARMBRUST et al (2009) identificaram possíveis obstáculos à adoção da computação em nuvens, dos quais se cita aqui os mais relevantes ao negócio:

- Disponibilidade do serviço: uma vez que o serviço é oferecido via Internet, pode ocorrer de a conexão entre a empresa cliente e o provedor falhar, seja por problemas na rede ou por indisponibilidade da empresa que fornece o serviço. Nesse caso naturalmente a empresa não teria acesso à aplicação SISESC e nem a seus dados e, para alguns negócios, o menor tempo sem operar representa prejuízos financeiros elevados;

- Segurança dos dados: este é um ponto bastante controverso nas discussões sobre cloud computing. Para qualquer solicitação de documentos armazenados nas nuvens ou qualquer operação realizada pelo sistema SISESC haverá o tráfego via Internet de dados da companhia. A dúvida é se já é possível garantir a total segurança dos dados em trânsito, sem riscos de vazamento de informações confidenciais da empresa;

- Confidencialidade e auditabilidade dos dados: os dados e informações da empresa estarão armazenados nos computadores do provedor do serviço, ao alcance de seus funcionários e colaboradores. Uma vez que o princípio é que ninguém acesse os dados de uma companhia a não ser ela mesma, a dúvida é como garantir que não haja quebra na confidencialidade. Mais do que isso a questão reside em como garantir que a empresa saiba se seus dados foram alterados ou visualizados, ou seja, como auditá-los. Nem todos executivos se sentem seguros sabendo que dados essenciais como sua situação financeira ou o projeto de um novo produto podem estar sendo acessados por quem não deveria;

- Congestionamento na transferência de dados: dependendo da quantidade de dados que se pretende transmitir, ou seja, da quantidade do serviço que se pretende utilizar, o custo pode ultrapassar os gastos com o modelo tradicional de uso de sistemas SISESC. Obviamente nesse caso trata-se de grandes corporações multinacionais, com muitas unidades de negócio espalhadas pelo mundo e que tenham ainda um grande fluxo de dados.

Outro desafio relevante é quanto aos provedores de serviço deste modelo computacional. Quando se fala em computação em nuvem não se pode esquecer que a relação que se estabelecerá entre este o provedor e a empresa contratante deve ser baseada no sistema colaborativo, onde o fornecedor deve ser um importante parceiro contribuindo para o sucesso ou fracasso do negócio. Nesse caso, é imprescindível que a empresa contratada tenha reputação ilibada e que tenha meios de conquistar e manter essa relação de confiança.

A empresa contratante não deverá se preocupar se o backup de seus dados está ou não sendo feito ou se haverá mudanças repentinas de código que possam prejudicar sua operação, por exemplo. Sendo assim, não se pode deixar de notar que a empresa que utiliza computação em nuvem tem forte dependência de seu provedor de serviços. Seus dados estão em posse da fornecedora, o que acarreta grande risco se ela resolver alterar seu sistema de cobranças arbitrariamente ou se não disponibilizar todas as informações 
de volta caso a empresa cliente opte por cancelar o contrato. Essa forte dependência é um dos aspectos que mais assustam as companhias quando pensam em adotar este modelo de negócio, e é o que mais retarda a disseminação dos serviços prestados.

A adoção da computação em nuvem também traz a possibilidade de revisão dos custos tangíveis e intangíveis na implantação e manutenção de sistemas. Embora seja inegável que esse paradigma da computação tenha potencial para alterar significativamente os orçamentos de TI e, por conseqüência, cooperar para que a empresa se foque em sua atividade fim, por causa das desvantagens aqui expostas ainda existem muitas dúvidas sobre a aplicabilidade real dentro do ambiente corporativo. Nem todos executivos confiam ainda plenamente nesse novo conceito e a percepção é que, por enquanto, seus receios suplantam o desejo de aproveitar estes benefícios.

\subsection{Infra-estrutura de computação em nuvem para o projeto proposto}

Para a criação da nuvem privada de que trata este projeto, utilizaremos duas máquinas, ambas rodando o sistema operacional Ubuntu 11.04. Uma funcionará como Front-end e a outra funcionará como um nó. Seguindo o passo a passo da instalação obtido na Eucalyptus Community, faremos as seguintes configurações:

\section{- Na máquina Front-end}

Nela instalaremos os pacotes Eucalyptus cloud (CLC), Eucalyptus walrus e Eucalyptus cluster controler(cc). O Cloud Controller (CLC) e o Walrus são componentes que ficam no nível mais alto, com um de cada em cada instalação do Eucalyptus. O controlador da nuvem é um programa feito em Java que oferece uma interface web para controle. Além de gerenciar requisições, o controlador de nuvem também faz o escalonamento de recursos e gerência de contas. O Walrus, também feito em Java, implementa um sistema de armazenamento que fica disponível interna e externamente à nuvem através de uma interface compatível com S3. O Cluster Controller(cc) é escrito em linguagem $\mathrm{C}$ e implantado como um serviço dentro do Apache onde cada cluster necessita de um controlador e ele é responsável pelo escalonamento de nível, pelo controle da rede e pelo controlador de armazenamento (SC -Storage Controler).

\section{- Instalando os pacotes -}

\$ sudo apt-get install eucalyptus-cloud eucalyptus-cc

\section{- Na máquina nó}

Nela instalaremos o pacote Eucalyptus node controller(nc), que funcionará como um controlador de nó, responsável por administrar as máquinas virtuais de seu nó.

\$sudo apt-get install eucalyptus-nc

Após instalarmos todos os pacotes, procedemos à configuração do servidor de cloud na máquina Front-end abrindo o navegador e digitar na barra de endereço o link https://localhost:8443. Será aberta uma janela. Depois de feito o login, cadastramos uma nova senha de acesso. Em seguida, clicamos no menu configuration e nele configuramos 
todos os parâmetros da nuvem, como o Cloud Host, o Walrus, o cluster e os tipos de máquinas virtuais que poderão ser instanciadas, conforme Figura 4.

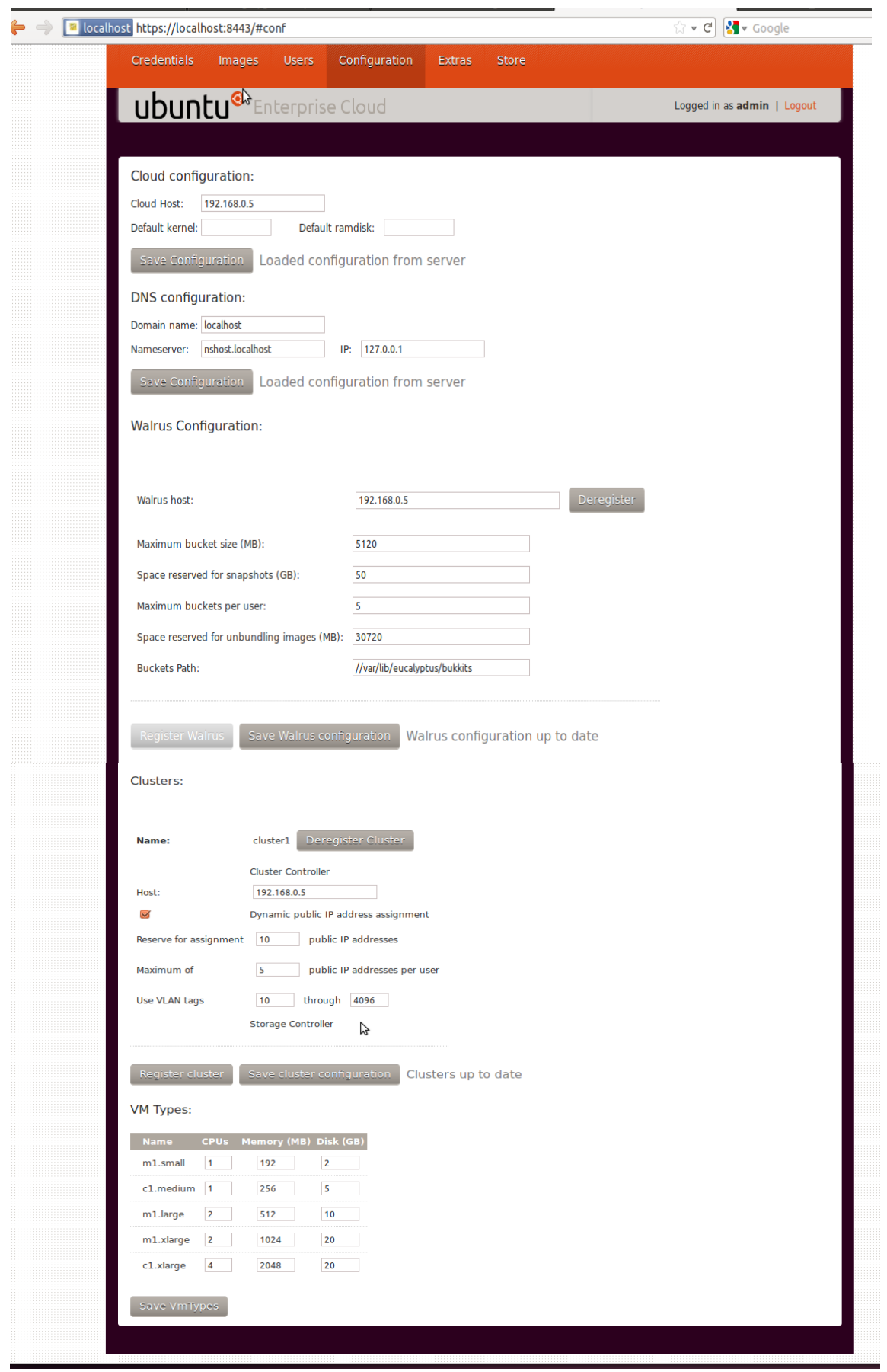

Figura 4. Página de configuração

Após a etapa de configuração do Cloud host, será necessária a instalação de certificados de segurança locais. Para isso, clicamos no menu Credentials e depois no botão Download Credentials. Será baixado o arquivo euca-admin-x509.zip que deverá ser desempacotado no diretório \$home/.euca e após isso deverá ser executado o comando, que dará acesso a vários serviços do Eucalyptus: \$ . /.euca/eucarc 
Neste momento, já poderemos adicionar os nós ao cluster já configurado. Para isso, digitamos o seguinte comando: \$ euca_conf --register-nodes <ip da máquina nó>

Como o Controlador da Nuvem conversa com os seus nós via SSH, registraremos as chaves de registro para que não hajam pedidos de senha entre eles. Registramos as chaves com os seguintes comandos: \$ sudo -u eucalyptus ssh-copy-id -i /var/lib/eucalyptus/.ssh/id_rsa.pub eucalyptus@<ip da máquina nó>

\section{- A seguir, com o comando}

\$ euca-describe-availability-zones verbose. Verificamos que toda a infra-estrutura entre o controlador e o nó está funcionando corretamente. O tipo da máquina virtual a ser instanciada vai depender dos recursos necessários para garantir um bom desempenho da nuvem. Para uso deste projeto, utilizaremos uma instância de uma máquina no tipo m1.small. Para disponibilizar imagens que serão administradas pela nuvem, na página de configuração, clicamos o menu Store e no botão Search. Serão listadas algumas imagens como uma pequena descrição de cada imagem e o botão Install (Figura 5)

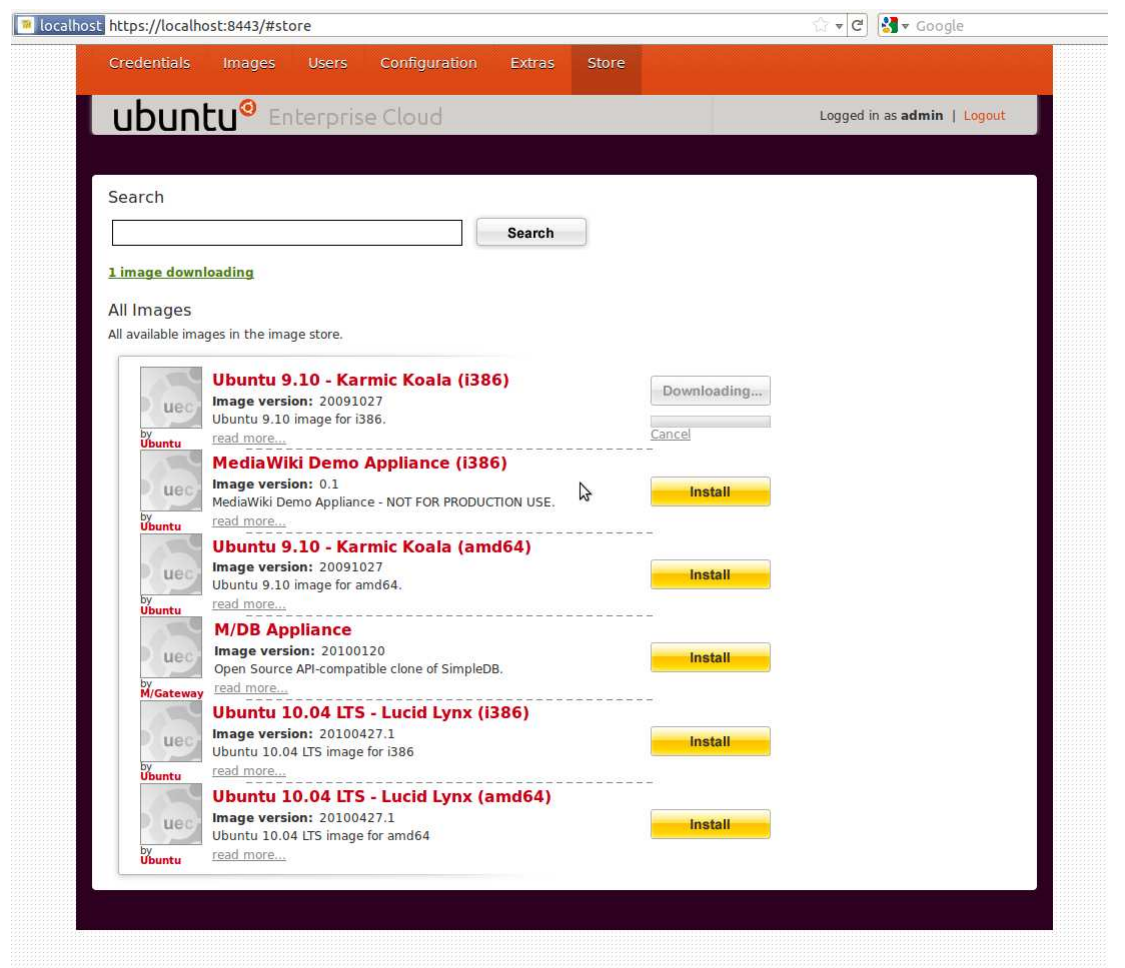

Figura 5. Página de download de imagens

Para a nuvem que trata este projeto, escolheremos a imagem Ubuntu 9.10 Karmic Koala (i386). Após o download da imagem, clicamos no link How to run? e será exibida as instruções para instancias a imagem em uma janela pop-up (Figura 6). 


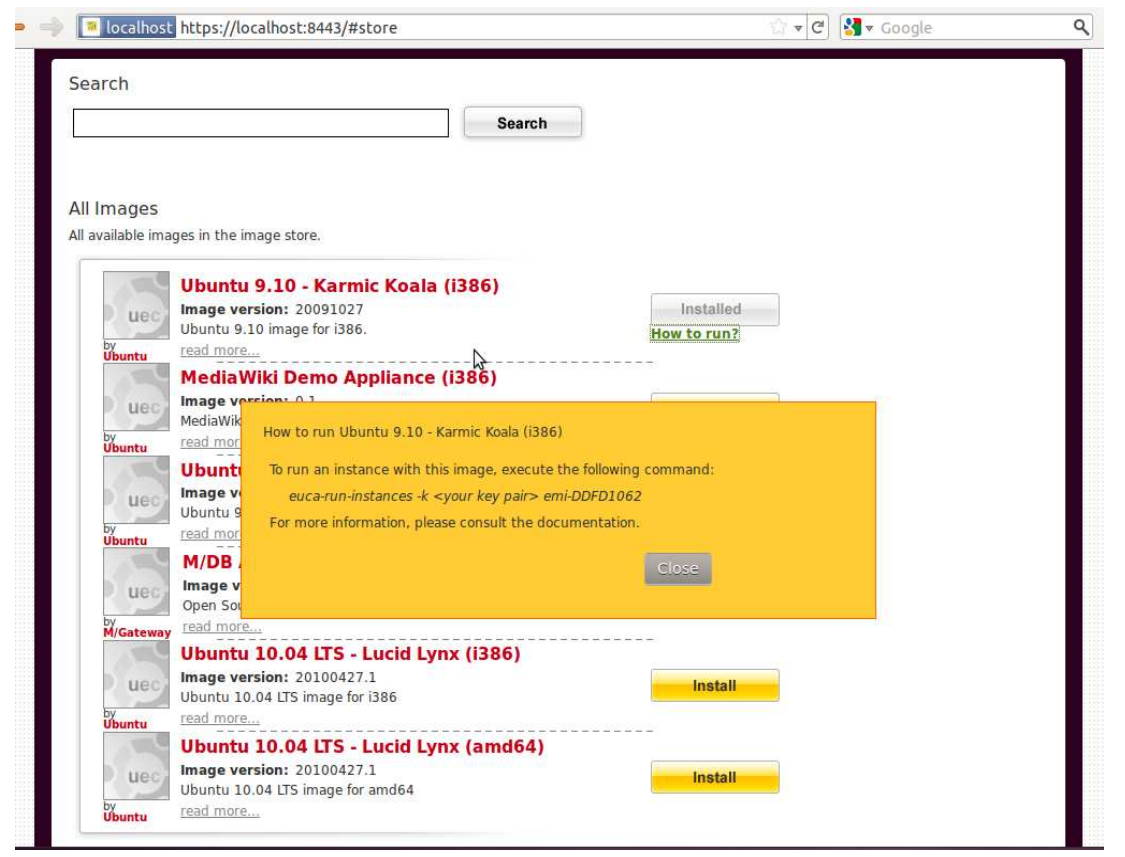

Figura 6. Informações para executar uma instância de imagem baixada

Tudo pronto agora para executar nossa máquina na nuvem. Quando executarmos a máquina, o objetivo é fazer login pelo shell seguro, ou SSH. Para isso será preciso gerar um par de chaves que são geradas com o seguinte comando:

Realizar certas operações em ambiente on-line de modo que os alunos e professores não precisem estar presentes na secretaria para realizarem tais tarefas.

\$ euca-run-instances -k chave -t m1.small emi-DDFD1062

Será apresentada informação e aguardaremos a instalação da instância e seu status mudar de pending para running. Depois disso, poderemos, enfim, conectar nossa máquina virtual na nuvem no IP indicado. Para isso acessamos através do SSH com o seguinte comando:

\$ ssh -1 root <ip da máquina instanciada>

Já poderemos criar a infra-estrutura para acesso ao SISESC. Instalaremos nessa nuvem recém-criada o servidor Apache, que servirá para disponibilizar o serviço http, o JDBC (Java Database Connectiviy), o banco de dados que será utilizado para a persistência dos dados e o código-fonte do SISESC.

\subsection{Protótipo SISESC}

SISESC é um software totalmente desenvolvido na linguagem Java e utiliza o JDBC para o armazenamento dos dados. $\mathrm{O}$ cliente em seu terminal acessa o sistema por meio de um browser opensource através da URL do sistema.

O Sistema de Gerenciamento da Rede de Ensino objetiva: 
- Permitir aos alunos emitirem boletim, verificarem histórico escolar e de pagamentos pelo sistema.

- Permitir que professores tenham acesso à listagem de turmas em que lecionam com os respectivos horários, além de poderem conferir as frequências dos alunos até o momento. Poderão adicionar ou alterar a frequência e as notas dos alunos durante o período anual.

- Permitir que funcionários da Secretaria registrem requerimentos e alterem o estado dos já existentes, além de poderem gerar relatórios a partir destes e encaminhar para direção, caso necessário. Poderão adicionar turmas a partir dos requerimentos da direção e gerar relatório com quadro de horários de turma e número de vagas.

- Quaisquer alterações de dados pessoais referentes ao aluno e todo o processo de matrícula do aluno no ano letivo, com a devida autorização do responsável, poderão ser realizadas pelo funcionário da secretaria, mediante solicitação através de requerimento.

- SISESC não se encarregará da organização de professores com suas respectivas disciplinas, turmas e horários; pois receberá a relação da Direção da Unidade.

- Agir como gestor financeiro, registrando a entradas e saídas no caixa da Unidade, bem como registrar os pagamentos efetuados e/ou não efetuados pelos alunos e emitir listagem de devedores mês a mês.

- Permitir que o administrador central da rede de escolas tenha total acesso a todas as unidades, bem como emitir relatórios e estatísticas de gestão personalizados.

Os diagramas de caso de uso e de classes estão apresentados como apêndice $1 \mathrm{e}$ 2 deste artigo. A interação dos quatro usuários finais - aluno, professor, secretaria e direção - com o SISESC, após a autenticação, ocorre a partir de opções constantes de menus disponíveis conforme apresentado na Figura 7.
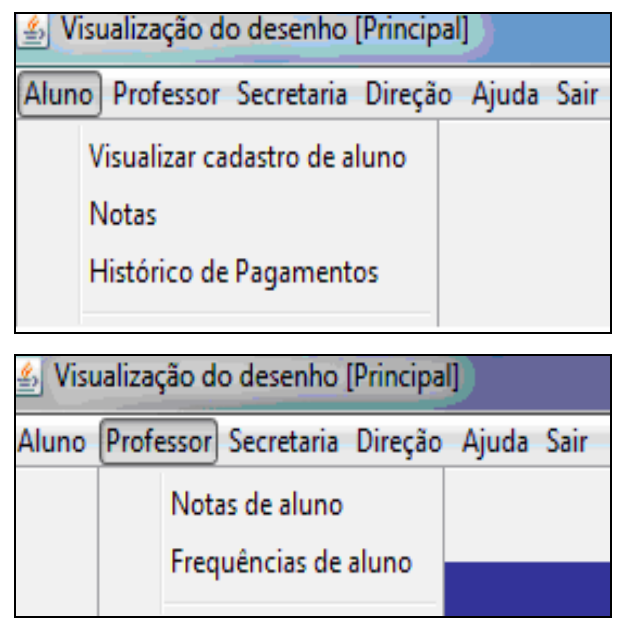
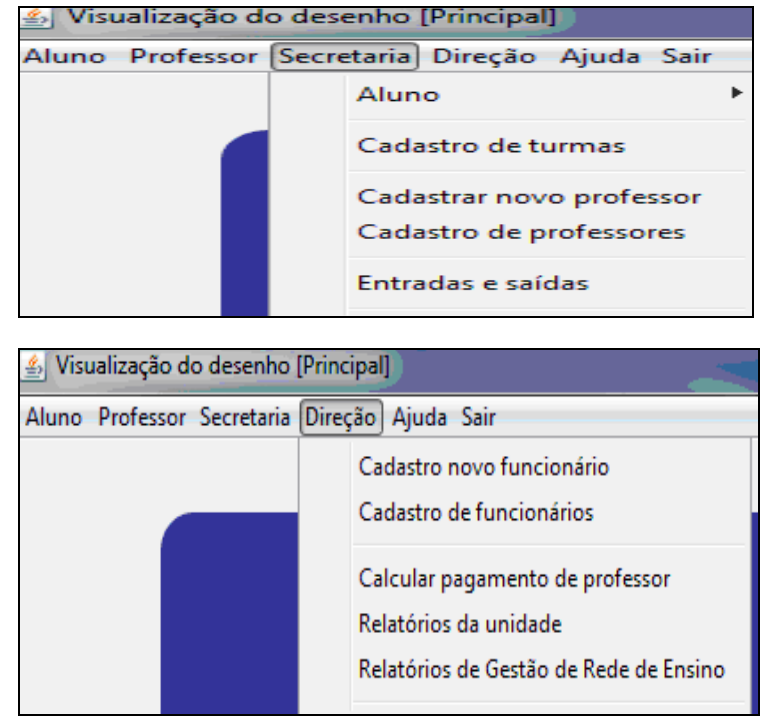

Figura 7. Menus da aplicação com detalhe para as opções: Aluno, Professor, Secretaria e Direção 


\subsection{Testes de Software em Computação em Nuvem}

Existem muitas propostas sobre a utilização da Nuvem como um ambiente de testes e alguns autores até já utilizam a nomenclatura TSaaS (Test Support as a Service). São duas as formas como os testes de softwares podem ser abordados na nuvem. A primeira diz respeito ao uso da Nuvem para testar softwares convencionais e dessa forma usufruir das características do ambiente da computação em nuvem. A segunda forma, e que mais interessa ao presente trabalho, é a realização de testes nos softwares implementados para serem oferecidos como um serviço na nuvem (SaaS).

Visualizamos o seguinte cenário de teste para nosso sistema. Ao fornecer ao cliente o SISESC, ele deverá ter passado por todas as etapas do ciclo de desenvolvimento de um sistema. Isso pode ser feito inicialmente da maneira convencional, já que o desenvolvedor pode trabalhar utilizando o PaaS (Plataform as a Service) e ele estará totalmente alheio a todas as características da Nuvem, utilizando o requisito da transparência em sistemas distribuídos que é o ponto forte do serviço PaaS. Porém é necessário saber como o sistema se comportará em situações reais e para isso ele deveria ser testado no local em que ficaria hospedado, isto é, a nuvem.

No entanto, existem poucas pesquisas que tratam dos testes em softwares implementados para serem oferecidos como um serviço na nuvem. A maior dificuldade dos testes em SaaS não está na falta de ferramentas para a sua realização, mas sim nos métodos formais efetivos para a realização de testes em ambientes de Nuvem. Para KING (2010), uma das poucas pesquisas que trata do assunto é o uso de AST (Autonomic Self-Testing) descrito em KING, (2007), em combinação com outra técnica de replicação das atualizações apenas depois das validações. Ou seja, o AST é uma forma de realizar testes em sistemas autônomos em que as atualizações não alterem o comportamento do ambiente, verificando se as atualizações ou as novas versões do SaaS possuem problemas para somente em seguida realizar a replicação das alterações em todo o ambiente da Nuvem. Pelo exposto, percebemos que uma das maiores dificuldades na computação em nuvem está na falta de modelos formais para a realização de testes de Softwares e com isso a dificuldade de se encontrar ferramentas para esse trabalho.

\section{Conclusões}

A computação como um serviço está emergindo e as empresas podem prestar serviços diretamente aos usuários por meio da Internet de acordo com as suas necessidades. Baseado nessa idéia, a computação em nuvem é um paradigma que está cada vez mais popular. Este trabalho apresentou um sistema de gerenciamento de unidade de ensino, por parte de uma escola, que assim como qualquer outra empresa, usando os conceitos de computação em nuvem, mostrando as vantagens oferecidas. Foi dada uma breve descrição do sistema, que para o usuário final é sistema como outro qualquer, dadas as características de transparência oferecidas pela nuvem. Alguns desafios de pesquisa ainda existem no contexto da computação em nuvem, porém as vantagens são superiores a esses desafios, principalmente no que tange a economia de recursos financeiros. 


\section{Referências Bibliográficas}

ARMBRUST, M., Fox, A., Griffith, R., Joseph, A.D., Katz, R.H., Konwinski, A., Lee, G., Patterson, D.A., Rabkin, A., Stoica, I., Zaharia, M. (2009) "Above the Clouds: A Berkeley View of Cloud Computing”, Tech. Rep. UCB/EECS-2009-28, EECS Department, University of California, Berkeley.

BUYYA, R. et al. (2008) "Market-Oriented Cloud Computing: Vision, Hype, and Reality for Delivering IT Services as Computing Utilities". In 10th IEEE international conference on high performance computing and communications.

FOSTER, I.; Yong, Z.; Raicu, I.; LU, S. (2008). Computação em nuvemand Grid Computing 360- Degree Compared. Department of Computer Science, University of Chicago.

GARTNER. Gartner Highlights Five Attributes of Cloud Computing. Disponível [Online] em: http://www.gartner.com/it/page.jsp?id=1035013, 2009. Acesso em: 13 de julho 2011.

IBM (2009a). Computação em nuvemfor the enterprise: Part 1: Capturing the cloud. Disponível em http://www.ibm.com/ developerworks/websphere/techjournal/0904_amrhein/0904_amrhein.html, Acesso em: 20 de junho 2011.

IBM (2009b). Cloud computing. Disponível em <http://www03.ibm.com/systems/information_infrastructure/ resources/ cloud/index.html. Acesso em: 20 de junho 2011.

KHAN, S., Maciejewsk, A., and Siegel, H. (2009) "Robust CDN Replica Placement Techniques”, In IEEE International Symposium on Parallel\&Distributed Processing.

LAMB, J. (2009). The Greening of IT: How Companies Can Make a Difference for the Environment. IBM Press.

MOTAHARI-NEZHAD, H. R.; STEPHENSON, B.; SINGHAL, S. (2009). Outsourcing Business to Computação em nuvemServices: Opportunities and Challenges. HP Laboratories. Disponível [Online] em: < http://www.hpl.hp.com/techreports/2009/HPL-2009-23.pdf>. Acesso em: 12 de julho 2009.

NURMI, D., Wolski, R., Grzegorczyk, C., Obertelli, G., Soman, S., Youseff, L., Zagorodnov, D. (2008) "Eucalyptus: A Technical Report on an Elastic Utility Computing Architecture Linking Your Programs to Useful Systems". University of California, Santa Barbara, October.

VAQUERO, L., Merino, L., Caceres, J.; Lindner, M. (2008) "A Break in the Clouds: Towards a Cloud Definition”, vol. 39, pp. 50-55, January.

King, T.M; Ramirez, A. E.; Cruz, R; Clarke, P. J. (2007). An integrated self-testing framework for autonomic computing systems, JCP, vol. 2, no. 9, pp. 37-49.

King, T.M.; Ganti, A.S. (2010). Migrating Autonomic Self-Testing to the Cloud,. 2010 Third International Conference on Software Testing, Verification, and Validation Workshops, April, pp. 438-443. 